\title{
Ética y conducta responsable en investigación: Una mirada a través de la Revista Médica Hondureña
}

\author{
Ethics and responsible conduct in research: A look through the Revista Médica Hondureña
}

Eleonora Espinoza, ${ }^{1}$ Jackeline Alger. ${ }^{2}$

${ }^{1}$ Médica, Maestría en Salud Pública;

${ }^{2}$ Médica, Doctorado $(\mathrm{PhD})$ en Parasitología.

Comité de Ética en Investigación Biomédica; Unidad de Investigación Científica; Facultad de Ciencias Médicas, UNAH; Instituto de Enfermedades Infecciosas y

Parasitología Antonio Vidal; Tegucigalpa.

RESUMEN. Antecedentes: La ética de la investigación tiene el fin de proteger los derechos, la integridad y la confidencialidad de los participantes en investigaciones. La integridad científica se refiere a la conducta responsable en la investigación. Objetivo: Describir la incorporación de la temática de ética de la investigación y la integridad científica entre las publicaciones de la Revista Médica Hondureña. Métodos: Análisis bibliométrico de las publicaciones en los números de cada volumen utilizando los términos investigación, integridad científica y ética (buscador temático http://www.bvs. hn/RMH/html5, revisión de los artículos publicados en la sección Ética y búsqueda temática por personal de la Biblioteca Médica Nacional). Resultados: De 54 artículos, en 28 (51.8\%), 19962019 , se hace referencia a la ética de la investigación y/o integridad científica. Hasta 2010, se habían publicado tres artículos. El tipo de artículo fue sección Ética 17, Editorial 5, Original 3, Opinión 2 y Especial 1. De acuerdo al área temática general, los artículos abordaron Promoción 2, Capacitación 2, Aplicación de los principios éticos de la investigación 16, Comités de ética en investigación 4 e Integridad científica 4. Discusión: A través de la Revista Médica Hondureña hemos visualizado los avances en investigación a través de la aplicación de buenas prácticas como lo es la ética de la investigación y la integridad científica. Ya que la publicación es el final del ciclo de la investigación, es necesario impulsar su fortalecimiento desde otros espacios de gestión. En Honduras existe la necesidad urgente de contar con un sistema nacional de investigación para la salud.

Palabras clave. Comités de ética en investigación, Ética en investigación, Ética en la publicación científica, Revisión de integridad científica.
Recibido: 17-03-2020; Aceptado para publicación 08-06-2020

Dirección para correspondencia: Dra. Eleonora Espinoza

Correo electrónico: ceibunahfcm@gmail.com

Declaración de relaciones y actividades financieras y no financieras y conflictos de interés: ninguna.

DOl: https://doi.org/10.5377/rmh.v88i1.11597

\section{INTRODUCCIÓN}

Desde la fundación de la Asociación Médica Hondureña en 1929 , sus asociados presentaban regularmente temas científicos en reuniones mensuales con el propósito de actualizar el conocimiento médico. ${ }^{1}$ Posteriormente se fueron desarrollando otros espacios para la divulgación científica oral y escrita. Uno de estos espacios es el propiciado por la Revista Médica Hondureña y otro es el Congreso Médico Nacional.

La Revista Médica Hondureña, como un medio de divulgación de la producción científica del gremio médico en particular y en el campo de la salud en general, registró su primera publicación en 1930. ${ }^{2,3}$ En 1934, se celebraron por primera vez las Jornadas Médicas, seguidas por las Segundas Jornadas Médicas en 1955, para dar origen a lo que ahora conocemos como el Congreso Médico Nacional. ${ }^{4}$ Para impulsar el desarrollo científico y estos espacios de divulgación científica, en 1954 se señalaba "... que sin servicios de estadísticas, sin equipos de laboratorio clínico adecuados, que sin departamentos de anatomía patológica, sin equipo de fotografía clínica, que sin la soltura que da al profesional su asistencia frecuente a reuniones nacionales e internacionales, no es posible estar en capacidad de codearse en un plan de igualdad con aquellos que si están disponiendo desde hace ya años de todos estos elementos..." ${ }^{5}$ Posteriormente y con la organización del Colegio Médico de Honduras, la Secretaría de Asuntos Educativos y Culturales y la creación del Centro de Educación Médica Continua (CENEMEC), los procesos de capacitación, investigación y divulgación científica se fueron sistematizando en un programa anual de educación médica continua que inició con la aprobación en la Asamblea General Ordinaria realizada en la ciudad de Tegucigalpa en el año 1982, de la creación de la Comisión Nacional de Educación Médica Continua y posterior creación de CENEMEC. ${ }^{6}$

La ética de la investigación es parte de las pautas internacionales que buscan garantizar la calidad en la producción científica en el diseño, la conducta, la ejecución, el seguimiento, la auditoría, el registro, el análisis y la comunicación de la 
investigación. La ética de la investigación específicamente contribuye a proteger los derechos, la integridad y la confidencialidad de los sujetos participantes en las investigaciones. ${ }^{7}$ Por otra parte, la integridad científica se refiere a la conducta responsable en la investigación y aborda temas como autoría responsable, mentoría, ciencia colaborativa y conflictos de interés. ${ }^{8}$ Las investigaciones científicas deben ser robustas desde el punto de vista de la metodología y la ética para ser consideradas de calidad. Con el objetivo de describir la incorporación de la temática de la ética de la investigación y la integridad científica entre las publicaciones de la Revista Médica Hondureña, se realizó un análisis bibliométrico de las publicaciones desde su primer número en 1930.

\section{MATERIALES Y MÉTODOS}

Se realizó un análisis bibliométrico de las publicaciones de la Revista Médica Hondureña para identificar la incorporación de los temas ética de la investigación y la conducta responsable en investigación o integridad científica. Se llevó a cabo una búsqueda utilizando los términos investigación, integridad científica y ética en el buscador temático incorporado en las páginas de la Revista Médica Hondureña en la Biblioteca Virtual en Salud de Honduras (http://www.bvs.hn/RMH/html5). También, se revisaron todos los artículos publicados en la sección de Ética de la Revista Médica Hondureña desde el inicio de dicha sección en el año 2011. Adicionalmente, se solicitó una búsqueda temática con esos términos al personal de la Biblioteca Médica Nacional. La búsqueda solamente incluyó los números de cada volumen, excluyendo los suplementos.

Los artículos identificados fueron revisados en todas sus secciones para constatar que en su contenido se referían a la ética de la investigación y/o a la conducta responsable en investigación o integridad científica. Una vez identificados los artículos de interés, se procedió a clasificarlos por año de publicación, tipo de artículos y tema específico. Adicionalmente se clasificaron de acuerdo a las siguientes áreas temáticas generales, identificadas por los autores en base a la temática específica: Promoción, Capacitación, Aplicación de los principios éticos de la investigación, Comités de ética en investigación e Integridad científica. Los resultados se presentan como frecuencias de las variables estudiadas. Algunos de los artículos identificados, se discuten en la sección de discusión por la relevancia de la temática abordada en la consecución del objetivo de este estudio.

\section{RESULTADOS}

Se identificó un total de 54 artículos publicados en la Revista Médica Hondureña de acuerdo a los términos investigación, integridad científica y ética, correspondientes al período 1943 a 2019. De éstos, en 28 (51.8\%) artículos se hace referencia al tema de la ética de la investigación y/o a la conducta responsable en la investigación y correspondieron al período 1996 a 2019.

En el Cuadro 1 se presenta una distribución de los artículos por año y de acuerdo al tipo de artículo, tema específico y área temática general. ${ }^{9 \cdot 36}$ En el período de 15 años, de 1996 a 2010, solamente se publicaron tres de los 28 artículos identificados. El resto se publicó entre los años 2011 y 2019. El tipo de artículo incluyó artículos de Ética 17, Editorial 5, Original 3, Opinión 2 y Especial 1. De acuerdo al área temática general, los artículos abordaron Promoción 2, Capacitación 2, Aplicación de los principios éticos de la investigación 16, Comités de ética en investigación 4 y la Integridad científica 4 (Cuadro 1).

\section{DISCUSIÓN}

A través de este análisis bibliométrico de las publicaciones sobre la temática de la ética de la investigación y la integridad científica en la Revista Médica Hondureña, hemos identificado que la primera referencia a los comités de ética en investigación se realizó en 1996 en un editorial. ${ }^{9}$ En los siguientes 15 años apenas hay dos referencias más a la ética de la investigación, otro editorial sobre la importancia del desarrollo de la investigación biomédica y la publicación en Honduras, ${ }^{10} \mathrm{y}$ un artículo de opinión sobre capacitación en planeación y evaluación efectivas de proyectos de investigación para la salud como un medio de promover la generación de investigación pertinente, ética y de calidad. ${ }^{11}$ Es así que, en Honduras, cuando en el mundo ya se contaba y se promovía el uso de una serie de herramientas y prácticas para la protección de los participantes humanos en investigaciones, ${ }^{7,16}$ la información era escasa como se ve reflejado en las pocas publicaciones sobre ese tema en la Revista Médica Hondureña.

A partir del año 2011, hubo un incremento en la publicación de los artículos sobre la temática de la ética en general y ética de la investigación en particular, relacionado con la creación de la sección de Ética en la Revista Médica Hondureña. En su editorial titulado La Revista Médica Hondureña a la vanguardia de la investigación científica, la Dra. Nelly Sandoval expresa que "...el actual consejo editorial se ha propuesto impulsar esta línea de trabajo, comenzando por promover confianza en la investigación científica en nuestro país, cumplir y hacer cumplir los requisitos de uniformidad internacional para manuscritos de revistas biomédicas, identificar, incentivar y asesorar al personal de salud interesado en este campo y al mismo tiempo abogar por el cumplimiento de las normas éticas en sus trabajos científicos." ${ }^{13}$ En la siguiente década las publicaciones se incrementaron y han variado ampliamente en su temática general, desde la promoción y la capacitación, hasta la aplicación de los principios de la ética de la investigación e integridad científica hasta los lineamientos para la conformación y desempeño de los comités de ética en investigación. Asimismo, la Revista Médica Hondureña, desde la página editorial, continúa contribuyendo a estos temas a través de la publicación científica apegada a los estándares internacionales de ética y calidad. ${ }^{35,36}$

En el año de 1967, un editorial se refiere a la investigación científica y docencia en la Facultad de Ciencias Médicas (FCM) UNAH, destacando el desarrollo alcanzado en la cátedra de ciencias fisiológicas y los estudios sobre plantas medicinales. ${ }^{37}$ Más de 40 años después, en un panel de discusión sobre la investigación en los Postgrados de Medicina celebrado en el 
Cuadro 1. Características de los artículos sobre ética de la investigación y conducta responsable en investigación de acuerdo al año, tipo de artículo y tema, Revista Médica Hondureña, 1996-2019, $n=28$.

\begin{tabular}{|c|c|c|c|c|}
\hline Año & $\begin{array}{l}\text { Tipo de } \\
\text { Artículo }\end{array}$ & Tematica Especifica & $\begin{array}{c}\text { Área Temática } \\
\text { General }^{*}\end{array}$ & Referencia \\
\hline 1996 & Editorial & 1. Política de investigación & 4 & 9 \\
\hline 2002 & Editorial & 2. Revista Médica Hondureña y la investigación biomédica & 3 & 10 \\
\hline 2010 & Opinión & 3. Capacitación en planeación y evaluación efectivas de proyectos de investigación para la salud & 2 & 11 \\
\hline \multirow[t]{6}{*}{2011} & Opinión & 4. Investigación en los Postgrados de Medicina UNAH & 3 & 12 \\
\hline & Editorial & 5. Revista Médica Hondureña y la investigación científica & 3 & 13 \\
\hline & Ética & 6. Reseña sobre el Congreso PRIM\&R 2010 & 1 & 14 \\
\hline & Ética & 7. Diez años del Comité de Ética en Investigación Biomédica (CEIB), Facultad de Ciencias Médicas, UNAH & 3 & 15 \\
\hline & Ética & 8. Reporte Belmont & 3 & 16 \\
\hline & Ética & 9. Registro de Ensayos Clínicos & 3 & 17 \\
\hline \multirow[t]{3}{*}{2012} & Ética & 10. Psiquiatría, ética e investigación en Honduras & 3 & 18 \\
\hline & Ética & 11. Principios de la ética de la investigación & 3 & 19 \\
\hline & Ética & 12. Publicación y autoría & 5 & 20 \\
\hline 2013 & Especial & 13. Health Research Web: una herramienta para la investigación & 3 & 21 \\
\hline \multirow[t]{3}{*}{2014} & Ética & 14. Práctica médica e investigación & 3 & 22 \\
\hline & Ética & 15. Integridad científica & 5 & 23 \\
\hline & Ética & 16. Celulas HeLa y dilemas éticos & 3 & 24 \\
\hline \multirow[t]{2}{*}{2015} & Ética & 17. The Global Health Network y capacitación en ética de la investigación & 2 & 25 \\
\hline & Ética & 18. Muestras médicas y guías médicas & 3 & 26 \\
\hline \multirow[t]{3}{*}{2016} & Original & 19. Características de la investigación en el Postgrado de Ginecología y Obstetricia & 3 & 27 \\
\hline & Original & 20. Aplicación consentimiento informado & 3 & 28 \\
\hline & Ética & 21. Reseña de Foro Global de Bioética en Investigación 2016 & 1 & 29 \\
\hline \multirow[t]{2}{*}{2017} & Ética & 22. Ética en la salud pública & 3 & 30 \\
\hline & Ética & 23. Composición de los Comités de Ética en Investigación & 4 & 31 \\
\hline \multirow[t]{3}{*}{2018} & Ética & 24. Conflictos de Interés y Comités de Ética en Investigación & 4 & 32 \\
\hline & Ética & 25. Competencia e Independencia de los Comités de Ética en Investigación & 4 & 33 \\
\hline & Original & 26. Trabajos investigación presentados en Congreso Médico Nacional & 3 & 34 \\
\hline \multirow[t]{2}{*}{2019} & Editorial & 27. Revista Médica Hondureña y estándares de publicación & 5 & 35 \\
\hline & Editorial & 28. Comunicación científica y conducta responsable en investigación & 5 & 36 \\
\hline
\end{tabular}

*Áreas temáticas generales: 1= Promoción, 2= Capacitación, 3= Aplicación de los principios éticos de la investigación, 4= Comités de ética en investigación, 5= Integridad cientíifica.

marco XVI Congreso de los Postgrados de Medicina, ${ }^{12}$ se señalaba la necesidad del apoyo estatal e institucional para formular, implementar y evaluar políticas y programas regionales, nacionales e institucionales de investigación en salud y participar en programas de cooperación técnica. Este potencial está aún por desarrollarse y especialmente en la ética de la investigación, ya que en Honduras no se cuenta con leyes y normativas en ese campo. Se siguen las normativas internacionales y es así como se crean comités institucionales por iniciativa propia cuyo fin último es velar por los sujetos participantes en investigaciones. En ese panel también se destacó el papel del Comité de Ética en Investigación Biomédica (CEIB) FCM UNAH, el cual fue creado en el año 2000.12, 15 Aunque en 1996 se promovía la creación de Comités de Ética en Investigación, en las publicaciones se ha dejado constancia de la lucha para mantener la autonomía e independencia de los Comités de Ética en Investigación cuando no hay apoyo institucional. ${ }^{31-33}$

La ética de la investigación persigue garantizar que las investigaciones en que participan seres humanos como sujetos de investigación se realicen respetando los tres principios éticos fundamentales: respeto a las personas, beneficencia y justicia, descritos en el Informe Belmont. ${ }^{16}$ El respeto a las personas destaca que los individuos deberán ser tratados como agentes autónomos y que las personas con autonomía disminuida tienen derecho a ser protegidas. La beneficencia va más allá de respetar las decisiones y proteger los sujetos de investigación de daños, sino también procurar su bienestar y acrecentar al máximo los beneficios. El principio de justicia se refiere a la distribución equitativa de los beneficios y potenciales riesgos de la investigación. ${ }^{16,19}$ En investigación estos aspectos están contemplados en lo que se denomina ética de la investigación, integridad científica y conducta responsable de los investigadores y su equipo, la cual trata sobre cómo deben comportarse los investigadores con aquellos sujetos que son voluntarios para participar en la investigación en particular y con la sociedad en general, incluyendo aspectos clave como el consentimiento informado, la confidencialidad, la privacidad, la comunicación privilegiada, el respeto y la responsabilidad. ${ }^{19,20,23}$

Cada vez hay un mayor reconocimiento a nivel internacional de que la investigación de calidad puede mejorar la salud, reducir la desigualdad y promover el desarrollo. ${ }^{38}$ Entre las publicaciones se encuentran varias de autoría o coautoría internacional que 
describen diferentes iniciativas como la Red Inter-Regional de Centros de Referencia para capacitación en cursos de planeación y evaluación efectivas de proyectos de investigación para la salud, ${ }^{11}$ el congreso anual de Responsabilidad Pública en Medicina e Investigación (PRIM\&R) ${ }^{14}$ el registro internacional de los ensayos clínicos, ${ }^{17}$ el Health Research Web como una herramienta para facilitar una mejor gobernanza de la investigación en América Latina y el Caribe ${ }^{21}$ la plataforma The Global Health Network para capacitación y colaboraciones ${ }^{25}$ y el Foro Global de Bioética en Investigación. ${ }^{29} \mathrm{~A}$ través de la investigación, los países pueden abordar problemas de salud complejos o emergentes, por ejemplo la actual pandemia de COVID-19, e identificar soluciones innovadoras. No obstante, la investigación en el área de salud pública se ha identificado como una de las funciones más débiles a pesar de ser fundamental para lograr sistemas de salud más eficientes y funcionales. . $^{30,39}$ Es necesario promover la ética de la investigación y la integridad científica entre profesionales y estudiantes de grado y postgrado en el campo de la salud. En vista de la limitante que representa la capacitación presencial, es importante contar con plataformas para la capacitación en línea como The Global Health Network (www.tghn.org), con la cual se cuenta desde el año $2014 .{ }^{25}$

En conclusión, por este recorrido de casi 25 años a través de la Revista Médica Hondureña, hemos visualizado los avan- ces y desarrollo de la investigación en Honduras a través de la implementación de buenas prácticas como lo es la ética de la investigación y la conducta responsable en la investigación, creación de comités de ética en investigación, la capacitación de los investigadores en buenas prácticas clínicas e integridad científica y promoción de la publicación responsable. Sin embargo, la publicación es la parte final del ciclo de la investigación por lo que es necesario impulsar su fortalecimiento desde otros espacios de gestión. En Honduras existe la necesidad urgente de contar con un sistema nacional de investigación para la salud eficazmente estructurado y bien gobernado para impulsar la investigación científica como parte de las soluciones a los problemas de salud del país.

\section{CONTRIBUCIONES}

Ambas autoras concibieron, diseñaron y llevaron a cabo el estudio. Ambas contribuyeron a la redacción del artículo, incorporaron las recomendaciones editoriales y aprobaron la versión final.

\section{AGRADECIMIENTOS}

Se reconoce y agradece el apoyo del personal de la Biblioteca Médica Nacional, Facultad de Ciencias Médicas, Universidad Nacional Autónoma de Honduras, Tegucigalpa, en la búsqueda de los artículos.

\section{REFERENCIAS}

1. Larios M. Historia de la Medicina Hondureña (Concluye). Rev Méd Hondur. 1934; 4 (46): 707-714.

2. Javier Zepeda CA. Reseña Histórica de la Revista Médica Hondureña 1930-1995. Rev Méd Hondur. 1995; 63(4):153-161.

3. Javier Zepeda CA. Reseña Histórica de la Revista Médica Hondureña 1930-2005. Rev Méd Hondur. 2005; 73(Supl 2):4-16.

4. Alger J, Alvarenga RE, Javier CA, Lopez Lutz E, Maradiaga E, Rivera O, et al. Reseña histórica del Congreso Médico Nacional y presentación del XLVI Congreso, Tegucigalpa, 2003. Rev Med Hondur. 2003;71:48-54.

5. Gómez-Márquez J Ante las Segundas Jornadas Médicas. Rev Méd Hondur. 1955; 23 (1): 823-825.

6. Colegio Médico de Honduras. Reglamento del Centro Nacional de Educación Medica Continua CENEMEC. La Gaceta. República de Honduras, 17 de Junio de 2000, Tegucigalpa, páginas 49-56.

7. Organización Panamericana de la Salud, Consejo de Organizaciones Internacionales de las Ciencias Médica. Pautas éticas internacionales para la investigación relacionada con la salud con seres humanos, $4^{\mathrm{a}} \mathrm{ed}$. Ginebra: CIOMS; 2016

8. Kalichman M. Responsible Conduct of Research Education (What, Why, and Does it Work?). Acad Med [Internet]. 2016[consultado 19 julio 2019]; 91(12): e10. Disponible en https://www.ncbi.nlm.nih.gov/pmc/articles/ PMC5378670/pdf/nihms824212.pdf

9. Medina MT [Editorial]. Política de investigación en las ciencias biomédicas en Honduras. Rev Méd Hondur. 1996; 64(3): 83

10. Varela Martínez CE [Editorial]. La Revista Médica Hondureña, la investigación biomédica y las alianzas estratégicas. Rev Méd Hondur. 2002; 70: 2.

11. Alger J, Gómez L, Jaramillo A, Saravia NG, Cuervo LG, Halpaap B. Red Inter-Regional de planeación y Evaluación Efectivas de Proyectos de Investigación para la Salud, Cali, Colombia, Abril 2010. Rev Méd Hondur. 2010;78(2):96-99.

12. Varela Martínez C, Sánchez J, Joya O, Padgett D, Reyes A. Conclusiones y recomendaciones del panel de discusión sobre la investigación en los Postgrados de Medicina, XVI Congreso de los Postgrados de Medicina, noviembre del 2010. Rev Méd Hondur. 2011; 79 (1): 33-34.

13. Sandoval NJ. La Revista Médica Hondureña a la vanguardia de la investigación científica. Rev Méd Hondur. 2011; 79 (2): 57.

14. Alger J, Padgett D. Responsabilidad Pública en Medicina e Investigación (PRIM\&R): Congreso Anual 2010 sobre Avances en Ética de la Investigación, San Diego, California. Rev Med Hondur. 2011; 79(1):40-42.

15. Espinoza E, Alger J, Padgett D, Gonzales M. Comité de Ética en Investigación Biomédica (CEIB) de la Facultad de Ciencias Médicas, Universidad Nacional Autónoma de Honduras: Experiencia 2000 - 2010. Rev Med Hondur. 2011; 79(2):98-102.

16. Reporte Belmont: Principios éticos y directrices para la protección de sujetos humanos de investigación. Rev Méd Hondur 2011; 79 (3): 140-145.

17. Reveiz $L$, Saenz $C$. El registro de ensayos clínicos en el marco del consentimiento informado: aplicando la Declaración de Helsinki. Rev Méd Hondur. 2011;79 (4): 216-217.

18. Espinoza E, Sosa Mendoza CA. Psiquiatría, ética e investigación en Honduras. Rev Méd Hondur. 2012;80 (130-32.

19. Principios de la ética de la investigación y su aplicación. Rev Méd Hondur. 2012; 80 (2): 75-76.

20. Publicación y autoría. Rev Méd Hondur. 2012;80 (4):179-182.

21. Villanueva EC, Ribeiro de Abreu D, Cuervo LG, Becerra-Posada F, Reveiz $L$, IJsselmuiden $C$. HRWEB: una herramienta para facilitar una mejor gobernanza de la investigación en América Latina y el Caribe. Rev Méd Hondur. 2013;81 (1):46-50.

22. Bustillo Urbina MC. Autonomía, ética y medicina. Rev Méd Hondur. 2014;82(2):78-80.

23. Espinoza E, Alger J. Integridad científica: fortaleciendo la investigación desde la ética. Rev Méd Hondur. 2014;82:126-128.

24. Portillo Vásquez SM. Las eternas células HeLa, el dilema ético de hoy. Rev Méd Hondur. 2014 82:164-167.

25. Alger J, Furtado T, van Loggerenberg F. The Global Health Network: Plataforma virtual para capacitación en ética de la investigación. Rev Med Hondur. 2015; 83(3-4):165-166. 
26. Lizardo Barahona JR. De la muestra médica a las guías médicas. Rev Méd Hondur. 2015;83:74-76.

27. Fuentes Barahona I, Pastrana K, Alger J. Caracterización de la investigación realizada en el Posgrado de Ginecología y Obstetricia, Facultad de Ciencias Médicas UNAH, 2012-2015. Rev Méd Hondur. 2016;84:6-11.

28. Guardado Williams RF, Guardado Williams CS, Valle Aguilar MP, Gómez Campos A, Padilla J, Cruz Nelson CA, Maradiaga E. Conocimiento y aplicación del consentimiento informado en pacientes del Hospital Escuela Universitario / Hospital Materno Infantil. Rev Méd Hondur. 2016; 84(3): 95100.

29. Alger J, Lescano R, Canario JA. Aspectos Éticos en la Investigación con Mujeres Embarazadas: Una Reunión del Foro Global de Bioética en Investigación, Buenos Aires, Argentina, 2016. Rev Méd Hondur. 2016;84:137139.

30. Fernández Vázquez JA. Etica en la salud pública de Honduras: la coyuntura actual. Rev Méd Hondur. 2017;85: 60-64.

31. Sierra M. La composición multidisciplinaria, pluralista y capacitada como factores clave en el funcionamiento de un comité de ética en investigación biomédica. Rev Méd Hondur. 2017; 85: 130-131.

32. Alegría A. La ética en los comités de ética en investigación: Conflicto de intereses. Rev Méd Hondur. 2018;86:71-72.

33. Alger J. Comités de ética en investigación: anotaciones sobre su competencia e independencia. Rev Méd Hondur. 2018;86:73-74.

34. Castejón Cruz OA, Núñez Savoff RA, Bush Wood SW. Características de los trabajos de investigación presentados en los congresos médicos nacionales de Honduras, 2007-2017. Rev Méd Hondur. 2018; 86: 113-118.

35. Alger J. [Editorial]. Fortalecimiento y mejora continua de la Revista Médica Hondureña. Rev Méd Hondur. 2019;87(1):5.

36. Alger J. [Editorial]. Comunicación científica: modelos actuales y evaluación de los investigadores. Rev Méd Hondur. 2019; 87 (2): 49.

37. Bermúdez Milla A [Editorial]. Comentarios. Investigación Médica en Honduras. Paridad Universitaria. Rev Méd Hondur. 1967;35:199-200.

38. Organización Panamericana de la Salud, Organización Mundial de la Salud. Política de Investigación para la Salud. 49a Consejo Directivo, 61a Sesión del Comité Regional CD49/10 (Esp.). [Internet]. Washington D.C.: OPS; 2009. [consultado 20 julio 2019]. Disponible en: https:/l www.paho.org/hq/images/stories/KBR/Research/politica $\% 20 \mathrm{de} \% 20$ investigacion\%20para\%20la\%20salud.pdf?ua=1

39. Organización Panamericana de la Salud, Organización Mundial de la Salud. La Salud Pública en las Américas. Nuevos conceptos, análisis del desempeño y bases para la acción. Washington D.C.: OPS; 2002. Publicación Científica y Técnica No. 589.
ABSTRACT. Background: Research ethics have the purpose to protect the rights, integrity and confidentiality of participants in research studies. Scientific integrity refers to responsible conduct in research. Objective: Describe the incorporation of the theme of research ethics and scientific integrity among the publications of the Revista Médica Hondureña. Methods: Bibliometric analysis of the publications in the numbers of each volume using the terms research, scientific integrity and ethics (thematic search at http://www.bvs.hn/RMH/html5, review of the articles published in the Ethics section and thematic search by staff of the National Medical Library). Results: Of 54 articles, in 28 (51.8\%), 19962019, reference is made to research ethics and/or scientific integrity. Until 2010, three articles were published. The type of article was section Ethics 17, Editorial 5, Original 3, Opinion 2 and Special 1. According to the general thematic area, the articles addressed Promotion 2, Training 2, Application of the research ethics principles 16, Research ethics committees 4 and Scientific integrity 4. Discussion: Through the Revista Médica Hondureña we have visualized advances in research through the application of good practices such as research ethics and scientific integrity. Since publication is the end of the research cycle, it is necessary to promote its strengthening from other management spaces. In Honduras there is an urgent need for a national health research system.

Keywords. Ethics committees, research; Ethics, research; Scientific integrity review, Scientific publication ethics. 